\title{
Cyclotron resonance in a cuprate superconductor
}

\author{
Danilo B. Romero \\ Physics Department, Virginia Tech, Blacksburg, Virginia 24061
}

(Received 24 April 1992)

\begin{abstract}
Anderson suggested that the temperature dependence of the normal-state Hall coefficient in the high$T_{c}$ superconductors comes from two distinct relaxation rates: a longitudinal relaxation rate $\left(\tau_{\mathrm{tr}}^{-1}\right)$ due to holon-spinon scattering and a transverse relaxation rate $\left(\tau_{H}^{-1}\right)$ due to spinon-spinon scattering. A generalized Drude model that incorporates these magnetic-field-induced anisotropy in charge-relaxation rates yields another possible signature of Anderson's conjecture: cyclotron resonance at $\omega=\omega_{c} \tau_{H} / \tau_{\mathrm{tr}}$, where $\omega_{c}$ is the cyclotron frequency. We discuss the feasibility of observing this new signature.
\end{abstract}

The observed temperature $(T)$ dependence of the normal-state dc resistivity $\left(\rho_{\mathrm{dc}}\right)$ and Hall coefficient $\left(R_{H}\right)$ of the high- $T_{c}$ superconductors demonstrates an apparent inconsistency that remains difficult to explain. $\rho_{\mathrm{dc}}$ increases linearly with $T, 1,2$ and far-infrared measurements $^{3-5}$ have shown unambiguously that a linear $T$ dependent scattering rate $\left(\tau^{-1}\right)$ is responsible for this behavior. Since $\rho_{\mathrm{dc}}=\left(m / n e^{2}\right) \tau^{-1}$, where $m$ is the effective mass and $n$ is the density of the charge carrier, the farinfrared results imply that the ratio $m / n$ must be constant. The inconsistency lies in the explanation of the Hall data based on a one-band Drude model. In this model, $R_{H} \propto\left(m / n e^{2}\right) \omega_{c}, \omega_{c}$ is the cyclotron frequency given by $\omega_{c}=e B / m$. Combined with the analysis of the resistivity ${ }^{1,2}$ and far-infrared data, ${ }^{3-5}$ the model suggests that $R_{H}$ must be $T$ independent, whereas experiments find $R_{H}$ decreasing with a rise in $T .{ }^{6}$ However, strong evidence $^{6}$ exists against other scenarios based on multiple Drude bands ${ }^{7}$ or magnetic skew-scattering ${ }^{8}$ mechanisms. Assuming that the one-band Drude model is correct, one is inevitably led to an unusual but interesting possibility: a $T$-dependent cyclotron mass that is distinct from the longitudinal transport mass.

Anderson ${ }^{9}$ proposed a certain class of strongly correlated one-band system in two dimensions (putatively claimed a Luttinger liquid) as the relevant model for the high- $T_{c}$ superconductors. Recently it was hypothesized ${ }^{10}$ that the observed temperature dependences of $\rho_{\mathrm{dc}}$ and $R_{H}$ are experimental signatures of the characteristic spincharge separation in this two-dimensional (2D) system. The spin-charge separation arises from an unusual interaction between the spinon ( $\pm \hbar / 2$ spin but no charge) and the holon ( $+e$ charge but no spin), a kind of "statistical interaction" due to an analogue of the Pauli restriction on the states occupied by an up spinon and a down spinon. ${ }^{11}$ This unconventional spinon-holon interaction is believed to be responsible for all of the observed anomalous normal-state properties of the high- $T_{c}$ superconductors. ${ }^{9}$ Spin-charge separation is not unique to Anderson's theory, however, as it also appears in gaugetheory models ${ }^{12,13}$ of the cuprates. What distinguishes Anderson's theory from the latter models is the behavior of the spinon in a Luttinger liquid. ${ }^{10,11}$ The spinon is similar to ordinary Fermion quasiparticles and forms a pseudo-Fermi surface. Anderson's explanation ${ }^{10}$ of the mutually inconsistent $\rho_{\mathrm{dc}}$ and $R_{H}$ data is based on the way an applied field couples to the spinon-holon interaction. In essence, an applied field is coupled to the spinon-holon interaction only if it changes the distribution and occupation of states in the vicinity of the spinon pseudo-Fermi surface. Such an effect is caused by an applied electric field (E) as it translates the spinon pseudoFermi surface. This effect leads to the coupling of $\mathbf{E}$ to the spinon-holon interaction. On the other hand, an applied magnetic field ( $B$ ) only causes a rotation of the spinon pseudo-Fermi surface so that $\mathbf{B}$ will not couple to the spinon-holon interaction. Consequently, a Luttinger liquid in crossed electric and magnetic fields should manifest an anisotropic transport behavior. Longitudinal transport (parallel to $\mathbf{E}$ ) properties will be governed by the unconventional spinon-holon scattering, while transverse transport (perpendicular to $\mathbf{E}$, due to $\mathbf{v} \times \mathbf{B}$ ) properties will be determined by a more conventional spinonspinon scattering (which is analogous to electron-electron scattering in a Fermi liquid). ${ }^{10}$

An outcome of this unique behavior of a Luttinger liquid in the presence of crossed electric and magnetic fields is an anomalous longitudinal relaxation rate $\left(\tau_{\mathrm{tr}}^{-1}\right)$, determined by holon-spinon scattering, that is distinct from a more conventional transverse relaxation rate $\left(\tau_{H}^{-1}\right)$, which is due to spinon-spinon scattering. Theoretical calculations ${ }^{14,13,12}$ and phenomenological models ${ }^{15}$ of the normal state of high- $T_{c}$ superconductors have shown that $\hbar \tau_{\mathrm{tr}}^{-1} \simeq \lambda k_{B} T$ and experiments ${ }^{3-5}$ yield $\lambda \simeq 2-3$. In analogy to Fermi-liquid theory, ${ }^{16}$ Anderson proposed ${ }^{10}$ that $\hbar \tau_{H}^{-1} \simeq\left(k_{B} T\right)^{2} / J+\hbar \tau_{m}^{-1}$, where $\tau_{m}^{-1}$ is the scattering rate due to static magnetic impurities and $J$ is the antiferromagnetic exchange energy which is Anderson's estimate for the spinon bandwidth. An incisive probe of the transverse relaxation rate is the $T$ dependence of the Hall angle $\theta_{H}\left(\cot \theta_{H} \propto \rho_{\mathrm{dc}} / R_{H}\right.$ in a one-band Drude model). Anderson's picture predicts a definite and experimentally verifiable signature of the $T$ dependence of $\cot \theta_{H}$. Note that in the conventional quasiparticle picture $\cot \theta_{H}=\left(\omega_{c} \tau_{\mathrm{tr}}\right)^{-1} \propto T$, while in Anderson's model $\cot \theta_{H}=\left(\omega_{c} \tau_{H}\right)^{-1} \propto T^{2}$. The evaluation of the Hall angle 
from recent Hall-effect measurements ${ }^{17,18}$ and calculations from previously published magnetotransport data ${ }^{2}$ confirmed the $T^{2}$ dependence of $\cot \theta_{H}$. Furthermore, the analysis succeeded in correlating the variation of the temperature dependence of $R_{H}$ found in various samples to the presence of static impurities. ${ }^{6}$

This new perspective of the normal-state magnetotransport in the high- $T_{c}$ superconductors led us to search for additional experimental signatures that would provide further corroboration to Anderson's conjecture. In this paper, we consider a model of a free carrier with distinct longitudinal and transverse transport properties in the presence of crossed electric and magnetic fields. We derive the ac-magnetoconductivity tensor $\sigma(\omega)$ from a phenomenological extension of the Drude model which incorporated these magnetic-field-induced anisotropic transport behavior. We arrived at an interesting new result: cyclotron resonance will be observed at $\omega=\omega_{c} \tau_{H} / \tau_{\mathrm{tr}}$. We discuss the feasibility of observing this novel feature.

In the Drude model, the equation of motion for a charge in the presence of an external electric and magnetic field is given by

$$
\tau \frac{d v_{i}}{d t}+v_{i}=\sum_{j=1}^{3} \mu \xi_{i j},
$$

where $\mu=e \tau / m$ is the mobility and $v_{i}$ is the $i$ th component of the velocity of the moving charge. For $\mathbf{B}=B \hat{z}$, the electric-field matrix $\xi_{i j}$ is given by

$$
\xi_{i j}=\left(\begin{array}{cc}
E_{x} & v_{y} B / c \\
-v_{x} B / c & E_{y}
\end{array}\right),
$$

which explicitly shows the longitudinal and transverseHall electric-field components. $\left(E_{x}, E_{y}\right)$ and $\left(v_{x}, v_{y}\right)$ are the $x$ and $y$ components of $\mathbf{E}$ and $\mathbf{v}$, respectively. Note that we intentionally excluded the $z$-axis components in Eq. (2) (and in all subsequent equations), since the main effect of the magnetic field is restricted within the $x y$ plane. We extend Eq. (1) to a model of a free carrier with distinct longitudinal and transverse transport properties by hypothesizing a mobility tensor,

$$
\mu_{i j}=e\left(\begin{array}{ll}
\tau_{\mathrm{tr}} / m_{\mathrm{tr}} & \tau_{H} / m_{H} \\
\tau_{H} / m_{H} & \tau_{\mathrm{tr}} / m_{\mathrm{tr}}
\end{array}\right),
$$

which represents the longitudinal and transverse transport mobilities of the free carrier. Defined this way, $\mu_{i j}$ allows for the possibility that the transport $\left(m_{\mathrm{tr}}\right)$ and cyclotron $\left(m_{H}\right)$ masses are also different. In the present phenomenological model, $\tau_{\mathrm{tr}}$ and $\mu_{i j}$ replaces $\tau$ and $\mu$, respectively, in Eq. (1). We emphasize that the subsequent term inside the summation in Eq. (1) is a product of the corresponding matrix elements of $\mu_{i j}$ and $\xi_{i j}$. This is imposed by the requirement that the extended model recovers Eq. (1) for $\tau_{\mathrm{tr}}=\tau_{H}$ and $m_{\mathrm{tr}}=m_{H}$. It is straightforward to check that these modifications to the Drude model mimic a magnetic-field-induced anisotropic transport behavior, which, as discussed above, Anderson claimed ${ }^{10}$ to be operational in the cuprates.

With an applied ac electric field, $\mathbf{E}(t)=\mathbf{E} e^{-i \omega t}$, and us- ing the well-known expressions for the current density, $\mathbf{J}=\boldsymbol{\sigma} \cdot \mathbf{E}=n e \mathbf{v}$, the longitudinal $\left(\sigma_{x x}\right.$ and $\left.\sigma_{y y}\right)$ and transverse $\left(\sigma_{x y}\right.$ and $\left.\sigma_{y x}\right)$ components of the ac conductivity tensor $\sigma$ are easily derived from Eq. (1) as

$$
\begin{aligned}
& \sigma_{x x}=\frac{\left(n e^{2} \tau_{\mathrm{tr}} / m_{\mathrm{tr}}\right)\left(1-i \omega \tau_{\mathrm{tr}}\right)}{\left(1-i \omega \tau_{\mathrm{tr}}\right)^{2}+\left(\omega_{c} \tau_{H}\right)^{2}}=\sigma_{y y}, \\
& \sigma_{x y}=\frac{\left(n e^{2} \tau_{\mathrm{tr}} / m_{\mathrm{tr}}\right)\left(\omega_{c} \tau_{H}\right)}{\left(1-i \omega \tau_{\mathrm{tr}}\right)^{2}+\left(\omega_{c} \tau_{H}\right)^{2}}=-\sigma_{y x} .
\end{aligned}
$$

For $\omega=0$, inserting the generally accepted linear- $T$ dependence of $\tau_{\mathrm{tr}}^{-1}$ (Refs. 3-5) and Anderson's suggested form ${ }^{10}$ for $\tau_{H}^{-1}$ in Eqs. (4a) and (4b) yields $T$-dependent expressions for $\rho_{\mathrm{dc}}$ and $R_{H}$ which are in agreement with magnetotransport measurements. Also, the dc results are, qualitatively, consistent with Anderson's model. ${ }^{10}$ Furthermore, it is easy to verify that for $\tau_{\mathrm{tr}}=\tau_{H}$ and $m_{\mathrm{tr}}=m_{H}$, Eqs. (4a) and (4b) reduce to expressions for the conventional Drude model.

The most interesting result is for finite frequency. For circularly polarized light, $E=E_{x} \pm i E_{y}$, the two modes that can propagate in the system are given by $\sigma_{ \pm}=\sigma_{x x} \pm i \sigma_{x y}$,

$$
\sigma_{ \pm}=\frac{i\left(n e^{2} / m_{\mathrm{tr}}\right)}{\omega \pm \omega_{c} \tau_{H} / \tau_{\mathrm{tr}}+i / \tau_{\mathrm{tr}}},
$$

where the minus and plus refer to cyclotron-resonanceactive (CRA) and cyclotron-resonance-inactive (CRI) modes, respectively. Equation (5) predicts that cyclotron resonance will be observed at $\omega=\omega_{c} \tau_{H} / \tau_{\text {tr }}$ with a width given by $1 / \tau_{\mathrm{tr}}$. One can possibly understand these results within the context of Anderson's theory ${ }^{10}$ by noting that the spinon by itself will not manifest cyclotron resonance, since it has no charge. Rather, it is the unusual spinonholon interaction that allows for the cyclotron motion of the spinon. Within this picture, the cyclotron lifetime must then be associated with $\tau_{\mathrm{tr}}$. The new cyclotron frequency can be viewed as arising from the renormalization of the spinon mass by the unconventional spinon-holon interaction, i.e., $m_{H}^{\prime}=m_{H} \tau_{\mathrm{tr}} / \tau_{H}$.

In general, $\tau_{\mathrm{tr}}$ and $\tau_{H}$ are both $T$ and $\omega$ dependent. Infrared $^{3,5,19}$ and transport ${ }^{1,2}$ measurements along with proposed models ${ }^{15,9}$ for the normal-state properties of the high- $T_{c}$ superconductors concurs that $\tau_{\mathrm{tr}}^{-1} \propto \max [\pi T, \omega]$. Following Anderson's suggestion ${ }^{10}$ that the transverse transport properties are due to Fermi-liquid-like scattering between spinons, $\tau_{H}^{-1} \propto \max \left[(\pi T)^{2}, \omega^{2}\right] / J .{ }^{16}$ We now discuss the feasibility of observing the new experimental signatures of cyclotron resonance derived from Eq. (5) at the two limiting cases. In the model calculations shown in Figs. 1 and 2, the dielectric function used to calculate the transmittance and reflectance is $\epsilon(\omega)=\epsilon_{\infty}+4 \pi i \sigma(\omega) / \omega$, where $\sigma(\omega)$ is given by Eq. (5). The parameters used were chosen from typical values suggested by resistivity ${ }^{1,2}$ and infrared ${ }^{3-5}$ data (for $\omega_{p}$ and $\lambda_{\mathrm{tr}}$ ), Raman measurements ${ }^{20}$ of the magnon peak (for $J)$, and the reported ${ }^{17,2}$ coefficient of the $T^{2}$-dependent term in $\cot \theta_{H}$ (for $\lambda_{H}$ ) of various high- $T_{c}$ superconductors. 


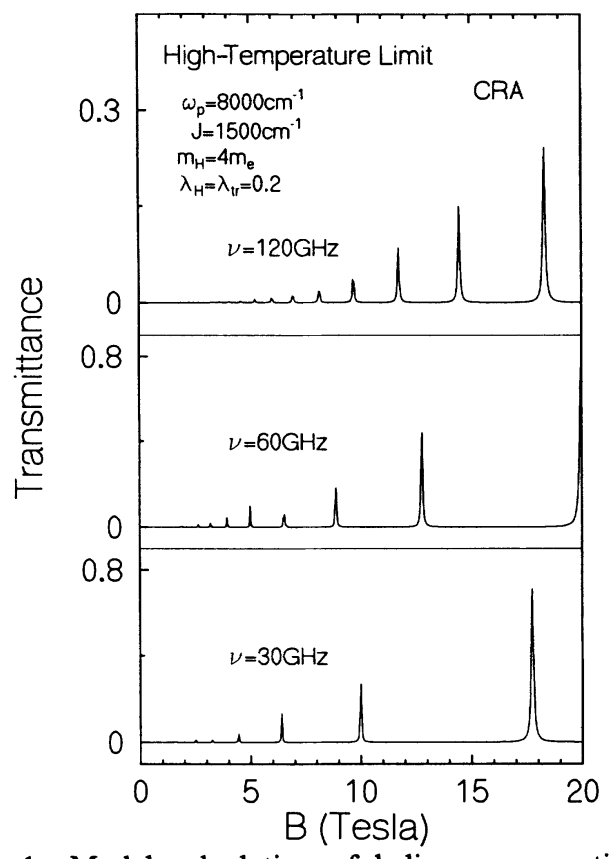

FIG. 1. Model calculation of helicon propagation in the high-temperature limit $(\pi T \gg \omega)$. The sample thickness used in the calculation is $0.1 \mathrm{~mm}$.

\section{A. High-temperature limit $(\pi T \gg \omega)$}

In this case, $\tau_{\mathrm{tr}}^{-1}=\pi \lambda_{\mathrm{tr}}(\pi T)$ and $\tau_{H}^{-1}=\pi \lambda_{H}(\pi T)^{2} / J$, where $\lambda_{\mathrm{tr}}$ and $\lambda_{H}$ are the coupling strengths of the spinon-holon and spinon-spinon interactions, respectively. Substituting these expressions for the two relaxation rates in Eq. (5), we find that cyclotron-resonance frequency is at $\omega_{\text {res }}=\omega_{c}\left(J \lambda_{\mathrm{tr}} / \pi \lambda_{H} T\right)$. Also, according to Eq. (5), the resonance condition is given by $\omega_{c} \tau_{H}=\omega_{c} J /\left[\pi \lambda_{H}(\pi T)^{2}\right] \gg 1$. Note that ordinarily cyclotron resonance in the cuprates will be difficult to detect, since the estimated cyclotron mass is a few times $m_{e}$, leading to values of $\omega_{c}$ at microwave frequencies and $\omega_{c} \tau_{H} \lesssim 1$. However, contrary to these conventional expectations, our new results suggest that $\omega_{c}$ (which is $\propto J / T$ ) and $\omega_{c} \tau_{H}$ (which is $\propto J / T^{2}$ ) are both rescaled to higher values at low temperatures $(T<<)$, perhaps due to the above-mentioned mass-renormalization effect. The possibility of observing cyclotron resonance in the cuprates at low temperatures was also pointed out by Chien et al. ${ }^{17,21}$ from their analysis of the Hall data.

The experimental condition $\omega<\pi T$ in this limit is favorable for observation of helicon propagation ${ }^{22}$ in the high- $T_{c}$ superconductors at very low frequencies. Model calculations for such helicon wave propagation in the cyclotron-resonance-active mode are shown in Fig. 1. The peaks are the dimensional helicon resonances that satisfy the Fabry-Perot interference condition $N \lambda / 2=d$, where $N$ is an integer, $d$ is the sample thickness, and $\lambda=2 \pi / \alpha$, with $\alpha \simeq \omega_{p}\left(\omega / \omega_{\text {res }}\right)^{1 / 2}$. In earlier studies of helicon propagation in semiconductors, ${ }^{23}$ the envelope function that determines the height of the different peaks depends strongly on the details of the relaxation mechanism. Therefore, helicon experiments may provide an alternative way of verifying the low-frequency relaxation processes in the high- $T_{c}$ superconductors.

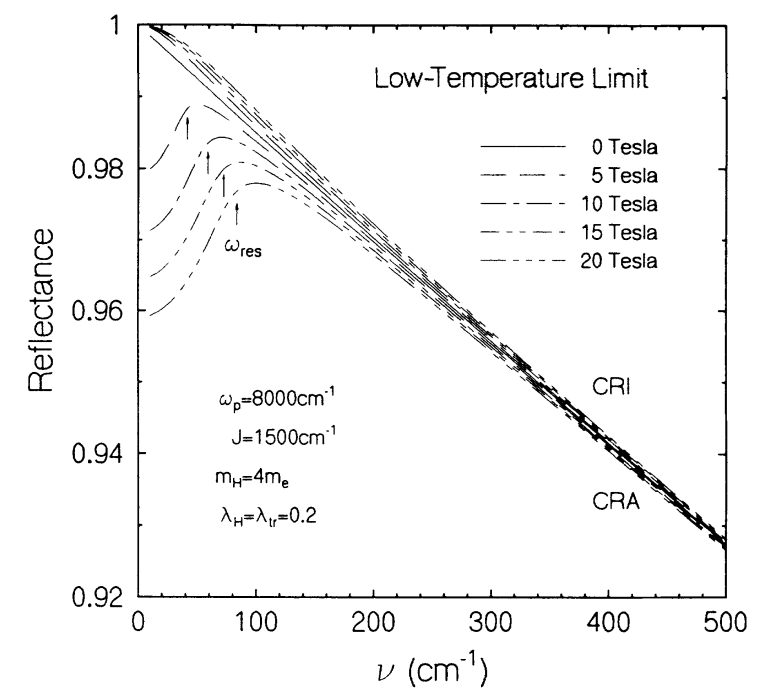

FIG. 2. Model calculation of the reflectance in the lowtemperature limit $(\pi T<<\omega)$. The arrows mark the position of $\omega_{\mathrm{res}}=\left(\omega_{c} J\right)^{1 / 2}$.

\section{B. Low-temperature limit $(\pi T \ll \omega)$}

In this case, $\tau_{\mathrm{tr}}^{-1}=\pi \lambda_{\mathrm{tr}} \omega$ and $\tau_{H}^{-1}=\pi \lambda_{H} \omega^{2} / J$. Equation (5) yields for the resonance frequency $\omega_{\text {res }}=\left(\lambda_{\mathrm{tr}} J \omega_{c} / \lambda_{H}\right)^{1 / 2}$. Aside from scaling the cyclotron energy to easily accessible far-infrared frequencies, the present result predicts a $B^{1 / 2}$ dependence of the resonance frequency, in contrast with the usual linear- $B$ dependence. The calculated reflectance shown in Fig. 2 demonstrates this behavior. Note the characteristic linear decrease in reflectance at zero magnetic field as the frequency is increased. With the magnetic field, a substantial absorption can be seen at low frequencies with an onset near $\omega_{\text {res }}$ for the cyclotron-resonance-active mode. For the cyclotron-resonance-inactive mode, the lowfrequency absorption is absent, and the onset of the linear drop in reflectance shifts upward with increasing magnetic field.

An ideal system for observing the novel effects predicted above is a clean cuprate superconductor with very low $T_{c}$. Model calculations, which include contributions due to static impurities, suggest that the above-mentioned features may be observed in samples with $T_{c} \leq 30 \mathrm{~K}$ and $\rho_{\mathrm{dc}}<10 \mu \Omega \mathrm{cm}$. Possible candidate systems are $\mathrm{Bi}_{2} \mathrm{Sr}_{2} \mathrm{CuO}_{6}$ and $\mathrm{La}_{2-x} \mathrm{Sr}_{x} \mathrm{CuO}_{4}$.

To conclude, we have examined in this work the consequences of a Drude model of a free carrier with distinct longitudinal and transverse transport properties induced by a magnetic field. We predict two definite experimental signatures of this model: (1) rescaling of $\omega_{c}$ to higher frequencies, and (2) $B^{1 / 2}$ dependence of $\omega_{\text {res }}$ in the lowtemperature limit. We speculate that these effects are due to the renormalization of the cyclotron mass by some unconventional interaction (perhaps the spinon mass by the spinon-holon interaction). Experimental verification of these cyclotron-resonance effects may provide indirect evidence of spin-charge separation in the high- $T_{c}$ superconductors. 
${ }^{1}$ M. Gurvitch and A. T. Fiory, Phys. Rev. Lett. 59, 1337 (1987).

${ }^{2}$ L. Forro, D. Mandrus, C. Kendziora, L. Mihaly, and R. Reeder, Phys. Rev. B 42, 8704 (1990).

${ }^{3}$ D. B. Romero, C. D. Porter, D. B. Tanner, L. Forro, D. Mandrus, L. Mihaly, G. L.Carr, and G. P. Williams, Phys. Rev. Lett. 68, 1590 (1992).

${ }^{4}$ K. Kamaras, S. L. Herr, C. D. Porter, N. Tache, D. B. Tanner, S.Etemad, T. Venkatesan, E. Chase, A. Inam, X. D. Wu, M. S. Hegde, and B. Dutta, Phys. Rev. Lett. 64, 84 (1990).

${ }^{5}$ J. Orenstein, G. A. Thomas, A. J. Millis, S. L. Cooper, D. H. Rapkine, T. Timusk, L. F. Schneemeyer, and J. V. Waszcazk, Phys. Rev. B 42, 1590 (1990).

${ }^{6}$ For a review, see N. P. Ong, in Physical Properties of High Temperature Superconductors II, edited by D. M. Ginsberg (World Scientific, Singapore, 1990).

${ }^{7}$ A. Davidson, P. Santhanam, A. Palevski, and M. J. Brady, Phys. Rev. B 38, 2828 (1988).

${ }^{8}$ A. T. Fiory and G. S. Grader, Phys. Rev. B 38, 9198 (1988).

${ }^{9}$ See P. W. Anderson and Y. Ren, in High Temperature Superconductivity, edited by K. Bedell et al. (Addison-Wesley, New York, 1989), and relevant references cited therein.

${ }^{10}$ P. W. Anderson, Phys. Rev. Lett. 67, 2092 (1991).

${ }^{11}$ P. W. Anderson, Phys. Rev. Lett. 65, 2306 (1990).

${ }^{12}$ L. B. Ioffe and P. B. Wiegmann, Phys. Rev. Lett. 65, 653
(1990).

${ }^{13}$ N. Nagaosa and P. Lee, Phys. Rev. Lett. 64, 2450 (1990).

${ }^{14}$ P. W. Anderson, Phys. Rev. Lett. 60, 132 (1988).

${ }^{15}$ C. M. Varma, P. B. Littlewood, S. Schmitt-Rink, E. Abrahams, and A. E. Ruckenstein, Phys. Rev. Lett. 63, 1996 (1989).

${ }^{16} \mathrm{D}$. Pines and P. Nozieres, The Theory of Quantum Liquids: Normal Fermi Liquids (Addison-Wesley, New York, 1966), p. 63.

${ }^{17}$ T. R. Chien, Z. Z. Wang, and N. P. Ong, Phys. Rev. Lett. 67, 2088 (1991).

${ }^{18}$ D. M. Ginsberg, in Proceedings of the International Conference on High $T_{c}$ Superconductors and Materials and Mechanisms of Superconductivity, edited by M. Tachiki (Kyoto, Japan, 1991).

${ }^{19}$ Z. Schlesinger, R. T. Collins, F. Holtzberg, C. Feild, S. H. Blanton, U. Welp, G. W. Crabtree, Y. Fang, and J. Z. Liu, Phys. Rev. Lett. 65, 801 (1990).

${ }^{20}$ P. E. Sulewski, P. A. Fleury, K. B. Lyons, S. W. Cheong, and Z. Fisk, Phys. Rev. B 41, 225 (1990).

${ }^{21}$ T. R. Chien, D. A. Brawner, Z. Z. Wang, and N. P. Ong, Phys. Rev. B 43, 6242 (1991).

22J. K. Furdyna, Appl. Opt. 6, 675 (1967).

${ }^{23}$ J. K. Furdyna, Phys. Rev. Lett. 14, 635 (1965). 